\title{
Phase II randomized trial of neoadjuvant metformin plus letrozole versus placebo plus letrozole for estrogen receptor positive postmenopausal breast cancer (METEOR)
}

Jisun Kim², Woosung Lim², Eun-Kyu Kim³ ${ }^{3}$ Min-Kyoon Kim, Nam-Sun Paik², Sang-Seol Jeong', Jung-han Yoon ${ }^{6}$, Chan Heun Park7, Sei Hyun Ahn', Lee Su Kim, Sehwan Han', Seok Jin Nam', Han-Sung Kang ${ }^{11}$, Seung II Kim², Young Bum Yoo ${ }^{13}$, Joon Jeong ${ }^{14}$, Tae Hyun Kim ${ }^{15}$, Taewoo Kang ${ }^{16}$, Sung-Won Kim ${ }^{17}$, Yongsik Jung ${ }^{18}$, Jeong Eon Lee ${ }^{10}$, Ku Sang Kim ${ }^{18}$, Jong-Han Yu' , Byung Joo Chae ${ }^{5}$, So-Youn Jung ${ }^{11}$, Eunyoung Kang ${ }^{17}$, Su Yun Choi ${ }^{19}$, Hyeong-Gon Moon ${ }^{4,20}$, Dong-Young Noh ${ }^{4,20}$ and Wonshik Han ${ }^{4,20^{*}}$

\begin{abstract}
Background: Neoadjuvant endocrine therapy with an aromatase inhibitor has shown efficacy comparable to that of neoadjuvant chemotherapy in patients with postmenopausal breast cancer. Preclinical and clinical studies have shown that the antidiabetic drug metformin has anti-tumor activity. This prospective, multicenter, phase II randomized, placebo controlled trial was designed to evaluate the direct anti-tumor effect of metformin in non-diabetic postmenopausal women with estrogen-receptor (ER) positive breast cancer.

Methods/Design: Patients meeting the inclusion criteria and providing written informed consent will be randomized to 24 weeks of neoadjuvant treatment with letrozole $(2.5 \mathrm{mg} /$ day) and either metformin ( $2000 \mathrm{mg} /$ day) or placebo. Target accrual number is 104 patients per arm. The primary endpoint will be clinical response rate, as measured by calipers. Secondary endpoints include pathologic complete response rate, breast conserving rate, change in Ki67 expression, breast density change, and toxicity profile. Molecular assays will be performed using samples obtained before treatment, at week 4, and postoperatively.
\end{abstract}

Discussion: This study will provide direct evidence of the anti-tumor effect of metformin in non-diabetic, postmenopausal patients with ER-positive breast cancer.

Trial registration: ClinicalTrials.gov Identifier NCT01589367

Keywords: Metformin, Letrozole, Neoadjuvant, Estrogen receptor-positive Breast cancer

\section{Background}

Metformin, which is commonly used to treat type 2 diabetes, is a relatively safe drug with known pharmacokinetics and manageable toxicities. In addition, numerous experimental, epidemiologic, observational, and clinical studies have shown that metformin has anti-

\footnotetext{
* Correspondence: hanw@snu.ac.kr

${ }^{4}$ Department of Surgery, Seoul National University Hospital, 101 Daehakro, Jongno-gu, Seoul 110-744, Korea

${ }^{20}$ Cancer Research Institute, Seoul National University College of Medicine, Seoul, Korea

Full list of author information is available at the end of the article
}

tumor effects [1]. For example, in a preclinical mouse xenograft model, metformin reduced the effective dosages of standard chemotherapeutic drugs and had preferential effects on tumorigenic cells [2]. A retrospective clinical study showed that patients taking metformin during neoadjuvant chemotherapy had a higher pathologic complete response (pCR) rate than diabetic patients not taking metformin or non-diabetes patients $(24 \%$ vs $8 \%$ vs $16 \%, \mathrm{p}=0.02)[3,4]$. Two potential mechanisms of anti-cancer action of metformin have been suggested. First, metformin may directly activate adenosine 
monophosphate kinase (AMPK), resulting in the downstream inhibition of mTOR signaling and the consequent suppression of cell proliferation [5-7]. Second, metformininduced decreases in circulating insulin and insulin-like growth factor (IGF) concentrations may reduce the activation of the IGF-receptor signaling axis, resulting in decreases in growth promotion and mitogenesis [8-10]. Thus, the anti-cancer effects of metformin are mediated through a systemic improvement in metabolic profile and by directly targeting tumor cells $[11,12]$.

Questions remain, however, about the clinical benefits of metformin as an anti-cancer agent in patients with breast cancer. Although one large-scale, phase III trial of adjuvant metformin has been initiated in women with breast cancer (NCIC CTG MA.32) [1], the accrual and treatment process is still ongoing, and several years of follow-up are needed to determine survival benefits. In addition, little is known about the effects of metformin on different subtypes of breast cancer or on the synergy between metformin and concurrently administered systemic agents. In addition, the optimal dosage of metformin that shows maximal anti-tumor effects with acceptable toxicities has not been determined. In this context, neoadjuvant treatment is the most efficient setting to assess the short-term in vivo effects of drug therapy in breast cancer patients. Neoadjuvant endocrine therapy results in a comparable response but lower toxicity compared with neoadjuvant chemotherapy in women with ER-positive breast cancer [13]. In postmenopausal women, aromatase inhibitors are associated with higher response rates than tamoxifen $[14,15]$. A recent phase II trial found that neoadjuvant treatment with everolimus, an mTOR inhibitor, plus letrozole resulted in a better response rate than letrozole alone [16]. In addition, neoadjuvant metformin was shown to lower Ki67 level [17]. These results suggested that metformin may be effective, when combined with letrozole, in postmenopausal women with ER-positive breast cancer. We therefore designed and initiated a phase II clinical trial evaluating the anti-tumor effect of neoadjuvant metformin in postmenopausal women with ER-positive breast cancer by comparing treatment with letrozole plus metformin or placebo.

\section{Methods/Design Study goal}

The goal of this study was to evaluate the benefits of combining metformin and letrozole in the neoadjuvant treatment of postmenopausal women with ER-positive breast cancer. The primary end point is the rate of tumor response (clinical response rate) at 24 weeks, determined by measurements with calipers; changes in size on ultrasound, mammography, and MRI will be used as secondary efficacy assessments. Clinical response includes complete response (CR) and partial response (PR) by RECIST v1.1 criteria.

\section{Study design}

METEOR is a phase II, prospective, randomized, doubleblinded, placebo-controlled multicenter clinical trial. Twenty-one centers belonging to the Korean Breast Cancer Society Study Group are participating in this study (KBCSG-013). Patients will be randomly assigned (1:1) to receive letrozole $2.5 \mathrm{mg} /$ day plus either metformin or placebo for 24 weeks before surgery. The initial dose of metformin will be $1000 \mathrm{mg} /$ day for the first week, followed by $1500 \mathrm{mg} /$ day for the second week, and $2000 \mathrm{mg} /$ day from the third week onward. Patients will be randomized sequentially, stratified by center, with randomization codes. Block randomization will be used (SAS 9.2) in cooperation with the Medical Research Collaborating Center (MRCC) of Seoul National University Hospital. All study personnel will be masked to treatment. This study protocol has been approved by the Korea Food and Drug Administration (KFDA) as well as the institutional review board of each center, and was registered at clinicaltrials.gov (NCT 01589367). Written informed consent was obtained from all participants.

\section{Eligibility criteria and sample size calculation}

Eligible patients are postmenopausal women with histologically confirmed ER-positive, stage II or III, primary breast cancer with palpable and clinically measureable tumors. The target population is 208 women, 104 in each arm. Sample size was calculated based on expected clinical response rates (cRR) of $55 \%$ in the letrozole plus placebo arm and $70 \%$ in the letrozole plus metformin arm, with $\alpha=0.10$ and $80 \%$ power and including an estimated $10 \%$ drop out rate. ER positivity is defined as $\geq 10 \%$ nuclear staining by immunohistochemistry or Allred score $\geq 3$. Criteria for determining menopause include bilateral oophorectomy, age $\geq 60$ years, or age $<60$ years and amenorrhea for $\geq 12$ months and FSH $>30 \mathrm{mIU} / \mathrm{ml}$. Patients with diabetes (HbA1c $\geq 6.5 \%$ or fasting plasma glucose (FPG) $\geq 126 \mathrm{mg} / \mathrm{dL}(\geq 7 \mathrm{mM})$ ); clinical T4, N3, or M1 disease; bilateral cancer, or inflammatory breast cancer will be excluded, as will patients with a history of lactic acidosis or at high risk of having metformin-induced lactic acidosis, such as those with high alcohol consumption or NYHA class III/IV congestive heart failure.

\section{Treatment schedule and evaluation}

Patients will be randomly assigned (1:1) to 24 weeks of neoadjuvant treatment with metformin plus letrozole or placebo plus letrozole (Figure 1). Medications will be distributed at each monthly visit, with compliance assessed by counting the remaining tablets.

Tumor size will be measured clinically with calipers before treatment and sequentially every month. Each patient will undergo a careful physical examination at each monthly visit, and patients with progressive disease (PD) 


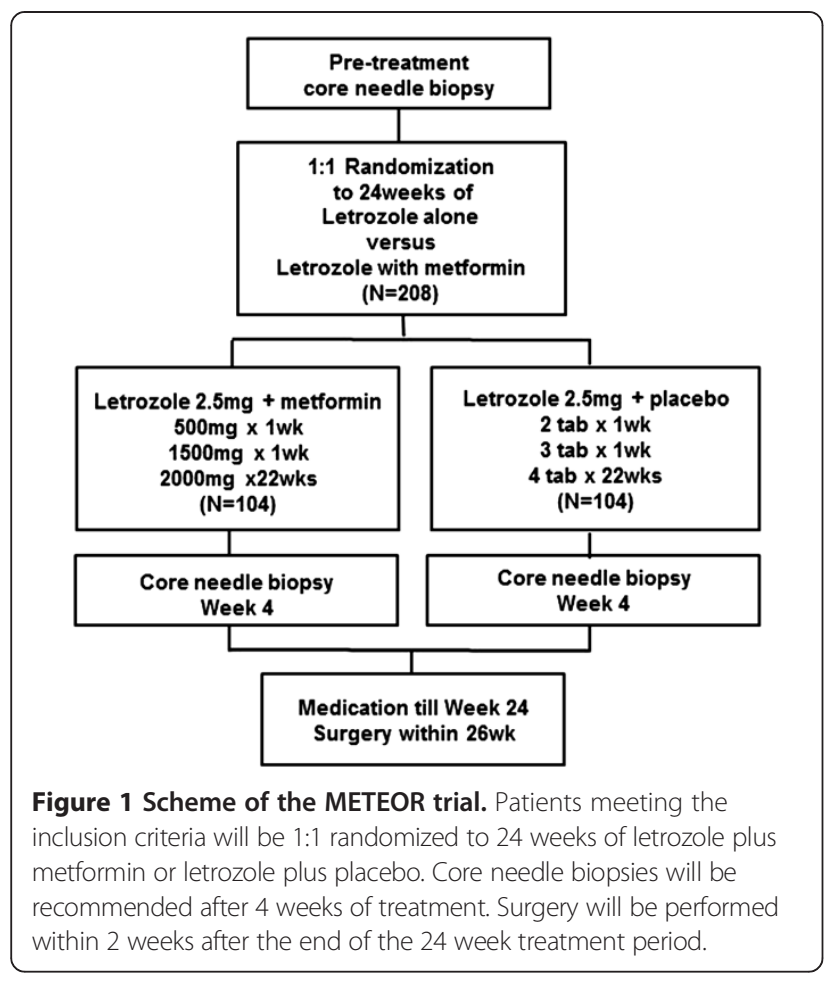

will be discontinued from the study and scheduled for immediate surgery. Patients with stable disease (SD) will be continued on treatment. Ultrasound-guided core needle biopsy samples will be obtained at the first visit and after four weeks of medication, with Ki67 levels in these samples centrally assessed. It is also recommended that 4-week blood samples be obtained to measure serum biomarker concentrations. Surgery will be scheduled within 2 weeks after completing the 24 weeks of medication. Tissue from surgical specimens will be collected for further planned assessments of biomarkers by paired analysis with the pretreatment and week 4 core needle biopsy specimens. HbA1c, FPG, insulin, c-peptide, and IGF-1 concentrations will be measured at baseline and after 12 and 24 weeks of treatment. Mammography, ultrasonography, and bilateral breast MRI will be performed before starting medication and after completion of treatment just before surgery.

\section{Analysis of the results}

The primary endpoint is cRR. Tumor response will be assessed by RECIST criteria v1.1. Tumor size will be measured by individual clinicians at monthly visits. The secondary endpoint is pathologic complete response (pCR), defined as the absence of invasive cancer at the primary site and in the axilla. Before treatment, each patients will be categorized by surgeons into one of three groups: 1) marginal for breast conservation, 2) candidate for mastectomy only, and 3) inoperable by standard mastectomy [18]. Baseline assessment and actually performed surgery will be compared. The breast conservation rate in each arm will be evaluated, and the toxicity profile of each arm will be assessed every 4 weeks using NCI-CTCAE version 4.0 (http://ctep.cancer.gov/reporting/ctc.html). All parameters will be collected and managed using an e-clinical trial platform $\left(\mathrm{MEBICA}^{\mathrm{mm}}\right)$.

\section{Translational research project}

In addition to assaying Ki-67, phosphorylated S6 kinase 1 (p-S6K1) will be analyzed by immunohistochemistry (IHC) in a central laboratory. Fresh frozen tissue of week 4 biopsies and final surgical specimens will be collected from several major hospitals participating in this study. These samples will be used for DNA microarray analysis or whole transcriptome sequencing using next generation sequencing (NGS) technology. Phosphorylation of AMPK(T172), expression of IR(insulin receptor) and OCT1(Organic cation transporter) will be measured. Assessment of apoptosis will be done by Miller-Payne Grading system along with various commercialized kits.

We previously reported that breast density reduction after short term adjuvant endocrine therapy was predictive of recurrence-free survival [19]. We intend to analyze the association between breast density reduction and response to endocrine therapy in this prospective trial. Pre- and post-medication mammography results and breast MRI images of all patients will be centrally reviewed. Breast density will be measured centrally using computer-assisted software, Cumulus (University of Toronto, Toronto Ontario, Canada), by a single observer. The density of both breasts will be assessed by breast MRI [20], with the cranio-caudal view of the contralateral breast used as a reference to evaluate the percent change in mammographic density.

\section{Discussion}

The maximum effective dose of metformin to treat hyperglycemia in patients with type 2 diabetes is $1000 \mathrm{mg}$ twice daily. However, the metformin dose that yields the maximal anti-tumor effect is unclear. Results from two xenograft models reported that the human equivalent of $1500-2250 \mathrm{mg} /$ day was needed to inhibit tumorigenesis [21-23]. A preoperative window of opportunity trial in breast cancer patients utilized a dose of $2000 \mathrm{mg} /$ day [24], whereas the ongoing NCIC CTG MA32 phase III clinical trial is testing the effect of adjuvant metformin, is utilizing a dosage of $1700 \mathrm{mg} /$ day. To use the maximum dose of metformin in this neoadjuvant setting, as well as to ensure patient safety, the dose is gradually increased over the first few weeks of treatment, and doses may be adjusted in response to toxicities/adverse events. The results of this trial will provide important information 
on the optimally effective and safest dose of metformin in non-diabetic breast cancer patients.

This study was designed to evaluate the direct antitumor effects of metformin in human breast cancer cancers. We expect that metformin will show a synergy with neoadjuvant letrozole in ER-positive breast cancer patients similar to that of the mTOR inhibitor, everolimus. Parallel translational research may provide a better understanding of the mechanism of action of metformin in cancer and may reveal biomarkers predictive of response to metformin.

\section{Abbreviations}

ER: Estrogen receptor; AMPK: Adenosine monophosphate kinase; pCR: Pathologic complete response; mTOR: mammalian target of rapamycin; IGF: Insulin-like growth factor; CR: Complete response; PR: Partial response; RECIST: Response evaluation criteria in solid tumors; CRR: clinical response rate; PD: Progressive disease; SD: Stable disease; MRI: Magnetic resonance image; p-S6K1: phosphorylated S6 kinase 1; NGS: Next generation sequencing.

\section{Competing interests}

All authors declare that they have no competing interests.

\section{Authors' contributions}

JK and WH drafted the manuscript and wrote the original protocol for the study. All authors participated in the design of the study. JK filed for ethical approvals from the KFDA and registered the trial on clinicaltrials.gov. WL performed the statistical analysis. EK designed the molecular study. MK was involved in the pharmaceutical process of SNUH and the process of patient enrollment. DN directed the entire process. All authors read and approved the final manuscript and are proceeding with the study in their respective centers.

\section{Acknowledgments}

This study is being supported by grant no 04-2012-0290 from the SNUH Research fund and by the National Research Foundation of Korea(NRF) grant funded by the Korea government(MSIP)(No. 2013005540).

Letrozole and metformin are being supplied by the pharmaceutical company, Shin Poong Pharm. Co., Ltd.

\section{Author details}

${ }^{1}$ Department of Surgery, University of Ulsan College of Medicine, Asan Medical Center, Seoul, Korea. ${ }^{2}$ Department of Surgery, Ewha Womans University School of Medicine, Seoul, Korea. ${ }^{3}$ Department of Surgery, Korea Cancer Center Hospital, Korea Institute of Radiological \& Medical Sciences, Seoul, Korea. ${ }^{4}$ Department of Surgery, Seoul National University Hospital, 101 Daehakro, Jongno-gu, Seoul 110-744, Korea. ${ }^{5}$ Department of Surgery, College of Medicine, The Catholic University of Korea, Seoul, Korea. ${ }^{6}$ Chonnam National University Hwasun Hospital, Chonnam National University Medical School, Hwasun, Korea. ${ }^{7}$ Department of Surgery, Breast and Thyroid Cancer Center, Kangbuk Samsung Hospital, Sungkyunkwan University School of Medicine, Seoul, Korea. ${ }^{8}$ Division of Breast and Endocrine Surgery, Hallym University Sacred Heart Hospital, Hallym University College of Medicine, Anyang, Korea. ${ }^{9}$ Department of Surgery, Inje University Sanggye Paik Hospital, Seoul, Korea. ${ }^{10}$ Department of Surgery, Samsung Medical Center, Sungkyunkwan University School of Medicine, Seoul, Korea. ${ }^{11}$ Center for Breast Cancer, National Cancer Center, Goyang, Republic of Korea.

${ }^{12}$ Department of Surgery, Yonsei University College of Medicine, Seoul, Korea. ${ }^{13}$ Department of Surgery, College of Medicine, Konkuk University, Seoul, Korea. ${ }^{14}$ Breast Cancer Center, Department of Surgery, Gangnam Severance Hospital, Yonsei University College of Medicine, Seoul, Korea. ${ }^{15}$ Department of Surgery, Inje University, Busan Paik Hospital, Busan, Korea. ${ }^{16}$ Busan Cancer Center, Department of Surgery, College of Medicine, Pusan National University, Busan, Korea. ${ }^{17}$ Department of Surgery, Seoul National University Bundang Hospital, Seoul National University College of Medicine, Seongnam, Korea. ${ }^{18}$ Department of Surgery, Ajou University School of Medicine, Suwon, Korea. ${ }^{19}$ Division of Breast and Endocrine Surgery, Kangdong Sacred Heart
Hospital, Hallym University College of Medicine, Seoul, Korea. ${ }^{20}$ Cancer Research Institute, Seoul National University College of Medicine, Seoul, Korea.

Received: 16 November 2012 Accepted: 3 March 2014

Published: 10 March 2014

\section{References}

1. Goodwin PJ, Stambolic V, Lemieux J, Chen BE, Parulekar WR, Gelmon KA, Hershman DL, Hobday TJ, Ligibel JA, Mayer IA, Pritchard KI, Whelan TJ, Rastogi P, Shepherd LE: Evaluation of metformin in early breast cancer: a modification of the traditional paradigm for clinical testing of anticancer agents. Breast Cancer Res Treat 2011, 126(1):215-220.

2. Hiopoulos D, Hirsch HA, Struhl K: Metformin decreases the dose of chemotherapy for prolonging tumor remission in mouse xenografts involving multiple cancer cell types. Can Res 2011, 71(9):3196-3201.

3. Jiralerspong S, Palla SL, Giordano SH, Meric-Bernstam F, Liedtke C, Barnett CM, Hsu L, Hung MC, Hortobagyi GN, Gonzalez-Angulo AM: Metformin and pathologic complete responses to neoadjuvant chemotherapy in diabetic patients with breast cancer. J Clin Oncol 2009, 27(20):3297-3302.

4. Garcia A, Tisman G: Metformin, B(12), and enhanced breast cancer response to chemotherapy. J Clin Oncol 2010, 28(2):e19. author reply e20.

5. Zhou G, Myers R, Li Y, Chen Y, Shen X, Fenyk-Melody J, Wu M, Ventre J, Doebber T, Fujii N, Musi N, Hirshman MF, Goodyear L, Moller DE: Role of AMP-activated protein kinase in mechanism of metformin action. J Clin Invest 2001, 108(8):1167-1174.

6. Hawley SA, Gadalla AE, Olsen GS, Hardie DG: The antidiabetic drug metformin activates the AMP-activated protein kinase cascade via an adenine nucleotide-independent mechanism. Diabetes 2002, 51(8):2420-2425.

7. Zakikhani M, Dowling R, Fantus IG, Sonenberg N, Pollak M: Metformin is an AMP kinase-dependent growth inhibitor for breast cancer cells. Cancer Res 2006, 66(21):10269-10273.

8. Pollak M: Insulin and insulin-like growth factor signalling in neoplasia. Nat Rev Cancer 2008, 8(12):915-928.

9. Goodwin PJ, Ligibel JA, Stambolic V: Metformin in breast cancer: time for action. J Clin Oncol 2009, 27(20):3271-3273.

10. Goodwin PJ, Pritchard KI, Ennis M, Clemons M, Graham M, Fantus IG: Insulin-lowering effects of metformin in women with early breast cancer. Clin Breast Cancer 2008, 8(6):501-505.

11. Bonanni B, Puntoni M, Cazzaniga M, Pruneri G, Serrano D, Guerrieri-Gonzaga A, Gennari A, Trabacca MS, Galimberti V, Veronesi P, Johansson H, Aristarco V, Bassi F, Luini A, Lazzeroni M, Varricchio C, Viale G, Bruzzi P, Decensi A: Dual effect of metformin on breast cancer proliferation in a randomized presurgical trial. J Clin Oncol 2012, 30(21):2593-2600.

12. Martin M, Marais R: Metformin: a diabetes drug for cancer, or a cancer drug for diabetics? J Clin Oncol 2012, 30(21):2698-2700.

13. Semiglazov VF, Semiglazov W, Dashyan GA, Ziltsova EK, Ivanov VG, Bozhok AA, Melnikova OA, Paltuev RM, Kletzel A, Berstein LM: Phase 2 randomized trial of primary endocrine therapy versus chemotherapy in postmenopausal patients with estrogen receptor-positive breast cancer Cancer 2007, 110(2):244-254.

14. Mlineritsch B, Tausch C, Singer C, Luschin-Ebengreuth $G$, Jakesz R, Ploner F, Stierer M, Melbinger E, Menzel C, Urbania A, Fridrik M, Steger G, Wohlmuth P, Gnant M, Greil R, Austrian Breast, Colorectal Cancer Study Group(ABCSG): Exemestane as primary systemic treatment for hormone receptor positive post-menopausal breast cancer patients: a phase II trial of the Austrian Breast and Colorectal Cancer Study Group (ABCSG-17). Breast Cancer Res Treat 2008, 112(1):203-213.

15. Eiermann W, Paepke S, Appfelstaedt J, Llombart-Cussac A, Eremin J, Vinholes J, Mauriac L, Ellis M, Lassus M, Chaudri-Ross HA, Dugan M, Borgs M, Letrozole Neo-Adjuvant Breast Cancer Study Group: Preoperative treatment of postmenopausal breast cancer patients with letrozole: a randomized double-blind multicenter study. Ann Oncol 2001, 12(11):1527-1532.

16. Baselga J, Semiglazov V, van Dam P, Manikhas A, Bellet M, Mayordomo J, Campone M, Kubista E, Greil R, Bianchi G, Steinseifer J, Mollow B, Tokaji E, Gardner H, Phillips P, Stumm M, Lane HA, Dixon JM, Jonat W, Rugo HS: Phase II randomized study of neoadjuvant everolimus plus letrozole compared with placebo plus letrozole in patients with estrogen receptor-positive breast cancer. J Clin Oncol 2009, 27(16):2630-2637. 
17. Goldenberg N, Glueck CJ, Loftspring M, Sherman A, Wang P: Metformindiet benefits in women with polycystic ovary syndrome in the bottom and top quintiles for insulin resistance. Metabolism 2005, 54(1):113-121.

18. Ellis MJ, Suman VJ, Hoog J, Lin L, Snider J, Prat A, Parker JS, Luo J, Deschryver K, Allred DC, Esserman L, Unzeitig GW, Margenthaler J, Babiera GV, Marcom PK, Guenther JM, Watson MA, Leitch M, Hunt K, Olson AJ: Randomized phase II neoadjuvant comparison between letrozole, anastrozole, and exemestane for postmenopausal women with estrogen receptor-rich stage 2 to 3 breast cancer: clinical and biomarker outcomes and predictive value of the baseline PAM50-based intrinsic subtype-ACOSOG Z103. J Clin Oncol 2011, 29(17):2342-2349.

19. Kim J, Han W, Moon HG, Ahn SK, Shin HC, You JM, Han SW, Im SA, Kim TY, Koo HR, Chang JM, Cho N, Moon WK, Noh DY: Breast density change as a predictive surrogate for response to adjuvant endocrine therapy in hormone receptor positive breast cancer. Breast Cancer Res 2012, 14(4):R102.

20. Nie K, Chen JH, Chan S, Chau MK, Yu HJ, Bahri S, Tseng T, Nalcioglu O, Su MY: Development of a quantitative method for analysis of breast density based on three-dimensional breast MRI. Med Phys 2008, 35(12):5253-5262.

21. Huang X, Wullschleger S, Shpiro N, McGuire VA, Sakamoto K, Woods YL, McBurnie W, Fleming S, Alessi DR: Important role of the LKB1-AMPK pathway in suppressing tumorigenesis in PTEN-deficient mice. Biochem J 2008, 412(2):211-221.

22. Anisimov VN, Berstein LM, Egormin PA, Piskunova TS, Popovich IG, Zabezhinski MA, Kovalenko IG, Poroshina TE, Semenchenko AV, Provinciali $M$, Re F, Franceschi C: Effect of metformin on life span and on the development of spontaneous mammary tumors in HER-2/neu transgenic mice. Exp Gerontol 2005, 40(8-9):685-693.

23. Anisimov VN, Egormin PA, Bershtein LM, Zabezhinskii MA, Piskunova TS, Popovich IG, Semenchenko AV: Metformin decelerates aging and development of mammary tumors in HER-2/neu transgenic mice. Bull Exp Biol Med 2005, 139(6):721-723.

24. Nathan DM, Buse JB, Davidson MB, Ferrannini E, Holman RR, Sherwin R, Zinman B, American Diabetes A, European Association for Study of D: Medical management of hyperglycemia in type 2 diabetes: a consensus algorithm for the initiation and adjustment of therapy: a consensus statement of the American Diabetes Association and the European Association for the Study of Diabetes. Diabetes Care 2009, 32(1):193-203.

doi:10.1186/1471-2407-14-170

Cite this article as: Kim et al:: Phase II randomized trial of neoadjuvant metformin plus letrozole versus placebo plus letrozole for estrogen receptor positive postmenopausal breast cancer (METEOR). BMC Cancer 2014 14:170.

\section{Submit your next manuscript to BioMed Central and take full advantage of:}

- Convenient online submission

- Thorough peer review

- No space constraints or color figure charges

- Immediate publication on acceptance

- Inclusion in PubMed, CAS, Scopus and Google Scholar

- Research which is freely available for redistribution 